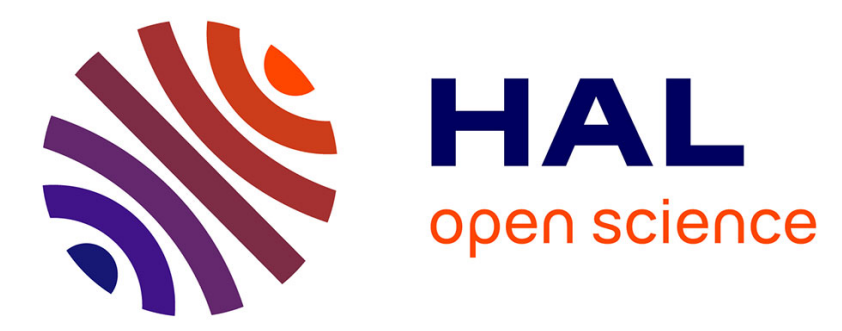

\title{
The Optimal Deployment of Recharging Stations for Electric Vehicles Based on Mobility Flows and Electric Grid Specifications
}

\author{
Abood Mourad, Martin Hennebel
}

\section{- To cite this version:}

Abood Mourad, Martin Hennebel. The Optimal Deployment of Recharging Stations for Electric Vehicles Based on Mobility Flows and Electric Grid Specifications. 2020 IEEE PES Innovative Smart Grid Technologies Europe (ISGT-Europe), Oct 2020, The Hague, Netherlands. 10.1109/ISGTEurope47291.2020.9248874 . hal-02958645

\section{HAL Id: hal-02958645 \\ https://hal.science/hal-02958645}

Submitted on 6 Oct 2020

HAL is a multi-disciplinary open access archive for the deposit and dissemination of scientific research documents, whether they are published or not. The documents may come from teaching and research institutions in France or abroad, or from public or private research centers.
L'archive ouverte pluridisciplinaire HAL, est destinée au dépôt et à la diffusion de documents scientifiques de niveau recherche, publiés ou non, émanant des établissements d'enseignement et de recherche français ou étrangers, des laboratoires publics ou privés. 


\section{The Optimal Deployment of Recharging Stations for Electric Vehicles Based on Mobility Flows and Electric Grid Specifications}

\author{
$1^{\text {st }}$ Abood Mourad \\ Laboratoire Génie Électrique et Électronique de Paris \\ CentraleSupélec, Université Paris Saclay \\ Gif-sur-Yvette, France \\ abood.mourad@centralesupelec.fr
}

\author{
$2^{\text {nd }}$ Martin Hennebel \\ Laboratoire Génie Électrique et Électronique de Paris \\ CentraleSupélec, Université Paris Saclay \\ Gif-sur-Yvette, France \\ martin.hennebel@centralesupelec.fr
}

\begin{abstract}
With the increasing interest in using electric vehicles (EVs) in future transportation systems, the need for deploying fast charging infrastructures becomes essential. In order to fulfil this need, it is important to anticipate EV future charging demands and requirements, and optimize their deployment. In this paper, we develop an optimization model to solve the problem of positioning fast-charging stations for EVs. The proposed model takes into account the different mobility flows and recharging demands as well as the constraints imposed by the available electric grid. In addition, the model considers the availability of alternative energy sources (i.e. photo-voltaic). For this purpose, we provide a mathematical formulation for the considered problem aiming at maximizing the covered recharging demand while respecting investment budget limits and the available capacities provided by the electric grid. Through a case study on ParisSaclay area, we obtain the optimal locations for deploying EV charging stations as well as the number of chargers that need to be installed at each charging station. Results also highlight the benefits of integrating locally-produced photo-voltaic energy on EV recharging service.
\end{abstract}

Index Terms - electric vehicles, recharging, optimization, mobility, electric grid

\section{INTRODUCTION}

Electric vehicles (EVs) have witnessed a considerable development in recent years. By improving their battery capacities and recharging technologies, EVs can circulate for long distances and be recharged in short periods of time (e.g. a Tesla Roadster can travel up to $340 \mathrm{~km}$ while recharging its battery takes no more than 20 minutes using fast-charging technology [1]). The need for deploying fast-charging infrastructures, especially on highways and high-speed roads, becomes essential. In order to fulfil this need and determine its required investments, it is important to anticipate EV future charging demands and requirements, and optimize their facility location taking into account the different mobility flows (i.e. small vehicles, lorry, etc.) and the existing electric grid [2].

In this paper, after reviewing the related literature (section III), we develop an optimization model to solve the problem of deploying recharging stations for EVs on a highway network

This research work is carried out as part of the PGMO research project at GeePs laboratory within CentraleSupélec engineering school. (section III). The proposed model takes into account the different mobility flows and recharging demands as well as the constraints imposed by the available electric grid. In addition, the model considers the availability of a local solar farm and integrates its produced energy to the system. More precisely, the deployment of EV charging stations and the number of fast-chargers is based on the charging demand in the first place. This deployment also respects the restrictions of the electric grid as well as the availability of parking places at charging stations. For this purpose, we provide a mathematical formulation for the problem aiming at maximizing the covered recharging demand while respecting investment budget limits and the available capacities provided by the electric grid (section IV). The relying optimization problem corresponds to the well-known facility location problem where our facilities are the recharging stations [3]. By solving this problem, we obtain the optimal locations for deploying EV charging stations as well as the number of chargers to be installed at each charging station (section $\mathrm{V}$ ). Finally, in section VI the key findings are highlighted and research perspectives are suggested.

\section{BACKGROUND}

Research on locating EV charging stations can be classified into three main categories; transportation, electric, and multidisciplinary approaches. Transportation approaches focus only on the transportation perspective (i.e. mobility flows and passengers demand) when designing EV charging networks [4]. On the other hand, electric approaches aim to locate EV charging stations in power systems such that their capacity and security requirements are satisfied and the investment costs needed to upgrade them are minimized [5]. As both transportation and electric perspectives are important, multidisciplinary approaches consider both types of constraints are considered [6]. In addition, different types of chargers have been considered in the literature. These are: level-1 chargers (referred to as slow charging, 110V/15A), level-2 chargers (220V/15-30A), and level-3 chargers (referred to as fast-charging, 400-500V/50A). These chargers have different 
features and requirements, such as; power capacities, charging times, cabling and outlets, etc. Some studies considered only one type of chargers while other studies integrated different types of chargers. For example, in [7], the distribution of level2 charging stations among territory segments is considered, based on the potential use of EVs and the different parking behaviors. In [6], the focus is on selecting locations for fast recharging stations (level-3) through a highway network for long-distance trips in the US. In [2], the deployment of both level-2 and level-3 chargers is considered to fulfil EV charging needs while respecting the specifications of the electric grid.

Regarding decision models, some studies suggested to use a Facility Location Model (FLM) [7], while others introduced a Flow Refueling Location Model (FRLM) as it provides a better coverage of mobility flows [4]. In addition, different objectives are assigned to these models, such as: minimizing investment costs [8], maximizing covered mobility flows or the number recharged EVs [2], or minimizing $\mathrm{CO}_{2}$ emissions [9]. Once the decision model is built along with its objectives and constraints, most studies suggest solving it using a mathematical solver (e.g. Cplex, Gurobi, etc.) so that the optimal deployment of EV charging stations is obtained. However, some studies developed other methods for solving these models, such as: Branch-and-Bound $(B \& B)$ and Dynamic Programming (DA) approaches (see [10] and [8] respectively). In this paper, we consider a multidisciplinary approach for locating EV fast charging stations. We also consider two types of fast chargers: for small vehicles and heavy trucks. In addition, we introduce a facility location model that aims at maximizing the covered charging demand. We then solve the proposed model using Cplex mathematical solver and analyze the obtained results through a case study on Paris-Saclay area.

\section{PROBlEM DESCRIPTION}

We focus in this problem on finding the optimal deployment of EV recharging stations and the number of fast chargers to be installed through a network of highways (mobility axes). In this problem, we consider two types of fast chargers, one for charging small electric vehicles, and the other for charging heavy electric trucks. In addition, We consider a set of potential charging locations $\mathcal{S}$ ( nodes $s 0, s 1, . ., s 4$ in Fig. 11. Every charging location $s \in \mathcal{S}$ is defined by a cost $\left(c_{s}\right)$ indicating required investment to use this location as a charging station, and has a maximum electric capacity $q_{s}$ and a max/min number of fast chargers that can be installed for small vehicles as well as heavy trucks $\left(\max _{s}^{a}, \min _{s}^{a}, \max _{s}^{b}\right.$ and $\min _{s}^{b}$ respectively). The investment cost $\left(c_{s}\right)$ includes land cost and cabling expenses. In addition, we consider a set of coupling nodes $N$, where these nodes are used to build our mobility paths (nodes $n 0, n 1, . ., n 5$ in Fig. 11. As such, we consider a set of paths $\mathcal{P}$ that link sequences of coupling nodes [11]. Every path $p \in \mathcal{P}$ is defined by its charging demands $d_{p}^{a}, d_{p}^{b}$, representing the number of EVs (i.e. vehicles and trucks respectively) to be recharged per day. The numbers of electric vehicles and trucks that can be recharged using a fast charger per day are defined as $\beta^{a}$ and $\beta^{b}$ respectively.

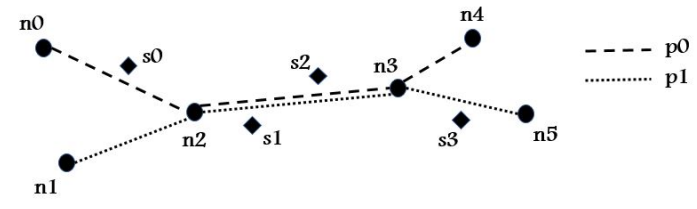

Fig. 1: Graph example - nodes and paths

Similarly, the amounts of electric power needed to recharge an electric vehicle or an electric truck using a fast charger are defined as $q^{a}$ and $q^{b}$ respectively. Moreover, installing a fast charger for electric vehicles or trucks requires additional costs (i.e. charger costs), these costs are defined as $c^{a}$ and $c^{b}$ respectively. Finally, we assume all electric vehicles to be identical, and thus, their recharging requirements to be the same (i.e. recharging any vehicle requires the same amount of time and energy). We consider the same assumption for electric trucks.

Hence, the choice of locating a recharging station at $s$ and the number of fast chargers to be installed must be based on the recharging demand through the path where $s$ is located. However, we can not install more chargers at a station than what the grid can accommodate. In addition, The number of chargers to be installed is also limited by the availability of places and parking slots at each recharging stations. As such, the aim is to build a model to optimize this deployment while the satisfied demand is maximized.

\section{Mathematical Model}

In order to model the problem, we introduce on binary variable:

$$
x_{s}= \begin{cases}1 & \text { if a charging station is deployed at node } s \in \mathcal{S} \\ 0 & \text { otherwise }\end{cases}
$$

In addition, we introduce a continuous variable $y_{p} \in[0,1]$ representing the demand coverage rate on path $p$, and two integer variables, $z_{s}^{a}$ and $z_{s}^{b}$, representing the number of fast chargers to be installed at charging location $s \in \mathcal{S}$ for electric vehicles and trucks respectively. The optimization model is thus formulated as follows:

$$
\begin{aligned}
& \operatorname{Max} Z=\sum_{p \in \mathcal{P}}\left(d_{p}^{a}+d_{p}^{b}\right) y_{p} \\
& \text { s.t. } \\
& \quad \sum_{s \in \mathcal{S}} c_{s} x_{s}+c^{a} z_{s}^{a}+c^{b} z_{s}^{b} \leq \mathcal{C} \\
& \sum_{p \in \mathcal{P}}\left(d_{p}^{a}+d_{p}^{b}\right) y_{p} \geq \mathcal{Q} \\
& \sum_{s \in \mathcal{S}_{p}} \beta^{a} z_{s}^{a} \geq d_{p}^{a} \quad \forall p \in \mathcal{P}
\end{aligned}
$$




$$
\begin{aligned}
& \sum_{s \in \mathcal{S}_{p}} \beta^{b} z_{s}^{b} \geq d_{p}^{b} \quad \forall p \in \mathcal{P} \\
& \min _{s}^{a} \leq z_{s}^{a} \leq \max _{s}^{a} \quad \forall s \in \mathcal{S} \\
& \min _{s}^{b} \leq z_{s}^{b} \leq \max _{s}^{b} \quad \forall s \in \mathcal{S} \\
& q^{a} z_{s}^{a}+q^{b} z_{s}^{b} \leq q_{s} \quad \forall s \in \mathcal{S} \\
& \begin{array}{l}
\text { if } y_{p}=0 \Longrightarrow z_{s}^{a}+z_{s}^{b}=0 \\
i f z_{s}^{a}+z_{s}^{b}>0 \Longrightarrow x_{s}=1 \\
\min _{s}^{a}, \max _{s}^{a}, \min _{s}^{b}, \max _{s}^{b} \in \mathcal{N} \\
x_{s}, y_{p} \in\{0,1\} \\
d_{p}^{a}, d_{p}^{b}, \beta^{a}, \beta^{b}, q_{s}, q^{a}, q^{b} \in \mathcal{N}, \forall s \in \mathcal{S} \\
z_{s}^{a}, z_{s}^{b} \in \mathcal{N} \\
\forall s \in \mathcal{S}, \forall p \in \mathcal{P}
\end{array} \quad \forall s \in \mathcal{S}, \forall p \in \mathcal{P} \\
& \forall s \in \mathcal{S}
\end{aligned}
$$

The objective function (1) maximizes the total covered charging demand based on the covered paths. Regarding model constraints, constraint (2) ensures that the sum of location costs and chargers installation costs does not exceed total budget limit. Constraint (3) states that a minimum of the overall charging demand must be satisfied. Constraints 4 and 5) ensure the satisfaction of charging demands for vehicles and trucks at each path. Constraints (6) and (7) state that the number of fast chargers (for vehicles and trucks) to be installed must be within the specified limits at each charging location. Constraints (8) ensure that the electric power required to operate the installed chargers does not exceed the available electric capacity at the charging locations. Constraints $(9)$ and (10) are used to link the variables introduced earlier in the model. Finally, constraints $(11)$ to $(15)$ define domains for the different elements of the model.

\section{Computational Results}

We present some results obtained by testing the proposed model using Cplex solver on a case study for Paris-Saclay area (section $\mathrm{V}-\mathrm{A}$ ). We also consider the availability of a photo-voltaic farm and highlight the benefits of integrating its generated power on EV recharging rates (section V-B.

\section{A. Paris-Saclay case study}

The interest in Paris-Saclay area is that it contains residential and business zones besides many transit axes that pass through it (e.g. A10, A6, N20 in Fig. 2). Many of these axes are not just used by personal and small vehicles, but also by heavy trucks carrying goods to nearby areas. Thus, these flows include local trips within the area (short-distance) as well as inter-city and international transit flows (long-distance).

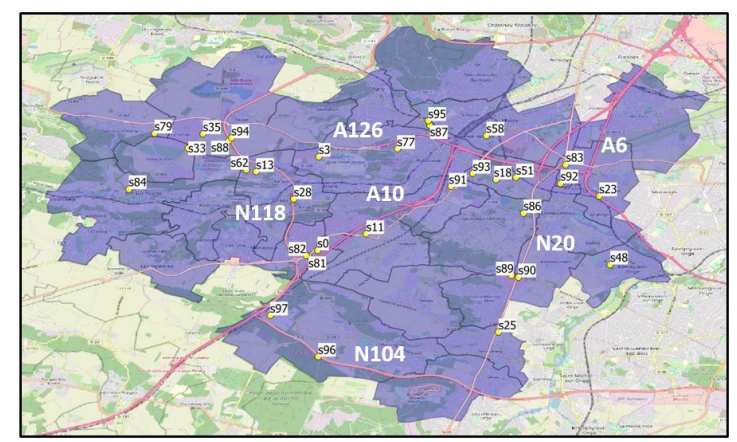

Fig. 2: Paris-Saclay - Major axes and recharging points

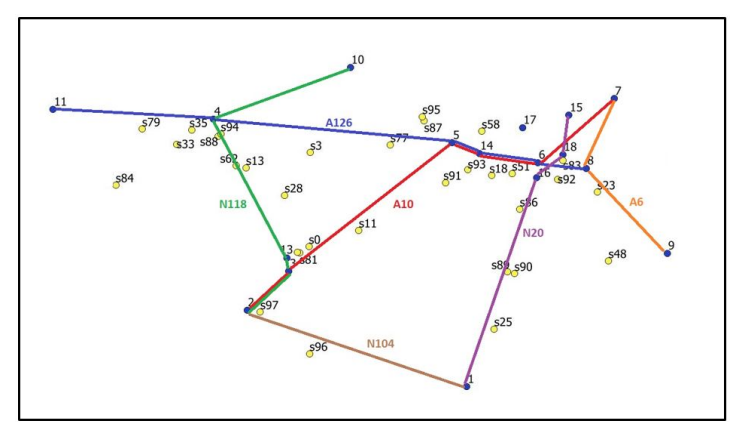

Fig. 3: Paris-Saclay - Mobility paths

As introduced earlier, we need to define a set of coupling nodes, a set of potential charging locations, and a set of mobility paths. Coupling nodes are defined by taking the intersections of major mobility axes in the area. Paths are then defined by sequences of these nodes. For example, in Fig. 3. the path "N118" is defined by the sequence of nodes $(2,3,13$, $4,10)$. We thus define 9 different paths representing EV flows and their recharging demands (Fig. 3). The actual number of electric vehicles and trucks to be recharged is then defined by taking $5 \%$ of the overall number of vehicles circulating through each path per day 1 . This percentage represents EV sales share of the overall vehicle market in France in 2020 [12]. Regarding charging locations, we consider the actual and projected charging stations, along with the actual service stations at Paris-Saclay area. Costs, electric capacities, and the maximum number of chargers that can be installed at each potential location are fixed based on their real dat: ${ }^{2}$ Charging

\footnotetext{
${ }^{1}$ Traffic data at Paris-Saclay area are provided by DiRIF (Road Direction in Ile-de-France region)

${ }^{2}$ Data of recharging stations and electric network specifications are provided by Paris-Saclay Agglomeration Community (CAPS)
} 
points are then associated to paths so that the model can decide which points to use and how many chargers to install based on their recharging demands (e.g. charging points: $s_{13}, s_{28}$ and $s_{62}$ are associated to path "N118"). We also assume that a fast charger requires $50 \mathrm{~kW}$ (20 minutes) to recharge an electric vehicle. In addition, fast chargers require $250 \mathrm{~kW}$ (30 minutes) to recharge an electric truck. We thus assume electric vehicles to be homogeneous in terms of battery and recharging time (same assumption for electric trucks). Finally, the costs of installing a fast charger for vehicles and trucks are estimated at $12 \mathrm{k}$ and $15 \mathrm{k}$ euros, respectively. Test parameters are summarized in Table \.

TABLE I: Test parameters

\begin{tabular}{|c|c|c|c|}
\hline Parameter & Value & Parameter & Value \\
\hline$c^{a}$ & $12 \mathrm{k}$ euros & $c^{b}$ & $15 \mathrm{k}$ euros \\
$\beta^{a}$ & 36 vehicles & $\beta^{b}$ & 24 trucks \\
$q^{a}$ & $50 \mathrm{~kW}$ & $q^{b}$ & $250 \mathrm{~kW}$ \\
\hline
\end{tabular}

Results of testing the proposed model on Paris-Saclay data are presented in Fig. 4. The number of fast chargers to be installed at each selected charging point is given in parenthesis (vehicle chargers, truck chargers). For example, the model suggests installing 6 vehicle chargers and 2 truck chargers at charging point $s_{13}$. We observe that the number of vehicle chargers is higher than that for trucks at all selected charging points. This is because the number of vehicles to be recharged is relatively higher at all defined paths, and thus requires more chargers. Results also indicate that the proposed solution can cover up to $33.1 \%$ of the overall charging demand. In the details, some paths are fully-covered (e.g. "A126" with $100 \%$ demand coverage), some are partially-covered (e.g. "A10" with $38 \%$ demand coverage), and others are poorly-covered (e.g. "A6" and "N104" with only $3.8 \%$ and $8.3 \%$ respectively). Low coverage rates can be explained by the small number of charging points that are associated to these paths and by the limited electric capacity at these points.

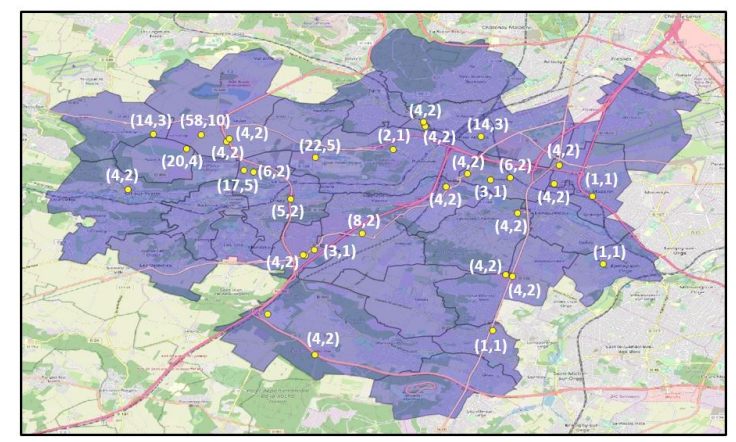

Fig. 4: Number of chargers per stations

Furthermore, we test the proposed model with different charging powers. We thus increase the charging power of chargers with up to $1.5,2$, and 4 times and we analyze its impact on covered charging demand. We observe that as chargers become more powerful, the demand coverage percentage increases (up to $40.9 \%, 52.5 \%$, and $71.1 \%$ respectively, Fig. 5). This highlights the importance of increasing the capacity of these chargers and thus integrating new sources of energy (e.g. photo-voltaic power) to accommodate this increase.

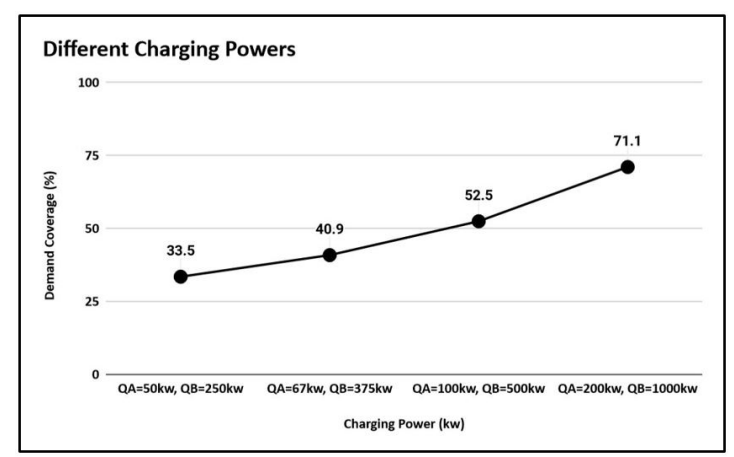

Fig. 5: Demand coverage with different charging powers

\section{B. Photo-voltaic power production}

Integrating green-energy sources can potentially increase electric network capacity while reducing its reliance on other polluting sources. For this purpose, a large photo-voltaic (PV) farm is to be installed at Paris-Saclay area (at point $s_{97}$, next to the intersection of "A10" and "N104" highways, Fig. 2 with 76500 solar panels and up to $24 \mathrm{GWh}$ annual power production. This farm can allow installing more EV chargers and increasing their recharging capacities. The generated power of a photo-voltaic farm at instant $t$ can be calculated as follows:

$$
P(t)=(1-\mu(t)) \times I(t) \times \delta \times \eta
$$

Where $\mu(t)$ is the cloudiness index at instant $t(\%), I(t)$ is the irradiation at instant $t\left(W / m^{2}\right), \delta$ is the surface of solar panels $\left(\mathrm{m}^{2}\right)$, and $\eta$ is the efficiency that corresponds to the electric quantity produced as a percentage of the received solar power. This generated power highly depends on irradiation and cloudiness index as they differ during seasons and day hours (e.g. PV production is maximal in a sunny day during Summer at noon). The actual values of irradiation at different seasons and day hours are fixed, and thus, known in advance $3^{3}$

Unlike the irradiation, the cloudiness index $(\mu(t))$ can not be fixed in advance as it highly depends on weather conditions (i.e. $\mu(t)$ is stochastic). We thus need to model this uncertainty using a set of scenarios and calculate their probability distribution. To overcome this difficulty, we consider 4 different day profiles: (1) Fully-Sunny, where $\mu(t)$ is between $0 \%$ \& 25\%, (2) Partially-Cloudy, where $\mu(t)$ is between $25 \%$ $\& 50 \%$, (3) Mostly-Cloudy, where $\mu(t)$ is between $50 \%$ \& $75 \%$, and (4) Fully-Cloudy, where $\mu(t)$ is between $75 \%$ \& $100 \%$. In addition, we divide the day into 8 time periods (i.e. (12AM,3AM), (3AM,6AM), .. , (9PM,12AM)). These day periods help in capturing different mobility patterns (e.g.

${ }^{3}$ We use data provided by the Photo-voltaic Geographical Information System (PVGIS) to build the irradiation matrix for Paris-Saclay area 
period (6AM-9AM) is a peak period when people commute, etc.). We also consider the four seasons (e.g. the probability of having a fully-sunny day is higher in Summer than in winter, Table III). As a result, we build our set of scenarios, where every scenario is defined by a day-profile, a day-period, and a season. In order to calculate their probabilities, we use weather measurements of the last 10 years and we cluster them according to day profiles, periods, and seasons using K-Means approach 4 The resulting probability distribution represents an input to our model.

TABLE II: Cloudiness index - Paris Orly (2010-2020)

\begin{tabular}{|c|c|c|c|c|}
\hline & Winter & Spring & Summer & Autumn \\
\hline Fully-Sunny & $16.9 \%$ & $27.2 \%$ & $35.6 \%$ & $22.9 \%$ \\
Partially-Cloudy & $6.7 \%$ & $9.1 \%$ & $15.6 \%$ & $9.1 \%$ \\
Mostly-Cloudy & $11.4 \%$ & $17.6 \%$ & $21.5 \%$ & $11.6 \%$ \\
Fully-Cloudy & $65.1 \%$ & $46.1 \%$ & $27.3 \%$ & $56.2 \%$ \\
\hline
\end{tabular}

In order to integrate PV production, an additional set of constraints need to be added to the mathematical model:

$$
q^{a} z_{v}^{a}+q^{b} z_{v}^{b} \leq P_{v} \quad \forall v \in \mathcal{V}
$$

Constraints (17) state that the energy required to operate the additional EV chargers must not exceed the amount of PV energy produced at PV farm $v$.

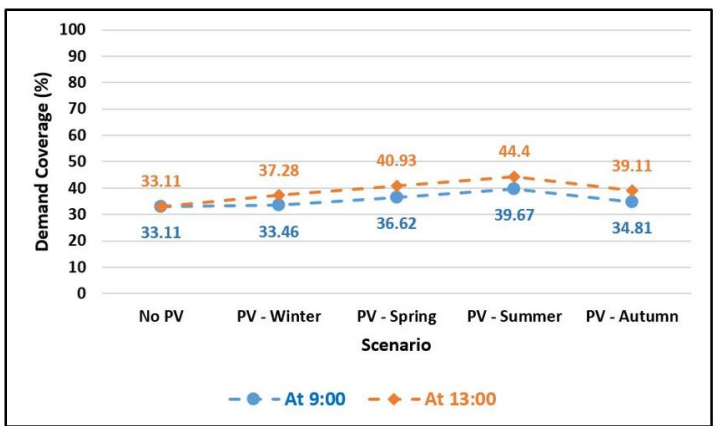

Fig. 6: PV production impact on demand coverage per season

To analyze the benefits of integrating PV energy, we quantify the amount of energy produced at different seasons and calculate the percentage of charging demand covered at each case. We then compare these rates to the original one where no PV production was considered. We consider two different demand flows: at 9AM (when vehicle and truck flows are maximal), and at 12AM (when solar irradiation and PV production are maximal) (Fig. 6). For both flows, and by averaging 10 different runs of the model, results indicate a positive effect of PV production on the overall demand coverage. This effect is most remarkable in Spring and Summer with approximately $7 \%$ to $10 \%$ increase on demand coverage. As the considered PV farm is located at the intersection of "A10" and "N104" highways, the demand coverage increases from $3.8 \%$ and $8.3 \%$

\footnotetext{
${ }^{4}$ Weather data for Paris-Orly airport, including cloudiness index, are provided by Météo France
}

to $28.9 \%$ and $42.6 \%$ at the morning peak (9AM) when PV production is included. Locally-produced PV energy can thus help in enhancing the quality of EV charging service while respecting the environmental aspects.

\section{CONCLUSIONS}

In this paper, an optimization model for deploying EV recharging stations through a network of highways has been introduced. The model has been tested using a Cplex solver and a case study on Paris-Saclay area has been performed. The obtained results has indicated the selected locations for EV recharging stations and highlighted the importance of improving chargers capacities and integrating PV production. One interesting perspective of this research is to consider the different energy production costs as they can change energy flows in the smart grid. We thus believe that this study helps in a better understanding of the potential deployment of EV recharging stations in real-life applications, and promoting more research towards studying this rising trend.

\section{ACKNOWLEDGMENT}

This research work is carried out within the PGMO research project at GeePs laboratory, CentraleSupélec. The work is in collaboration with the Institute of Research and Technology SystemX and the Agglomeration Community of Paris-Saclay.

\section{REFERENCES}

[1] H. Shareef, MD. Mainul Islam, and A. Mohamed, A review of the stageof-the-art charging technologies, placement methodologies, and impacts of electric vehicles, Renewable and Sustainable Energy Reviews, vol. 64, pp. 403-420, 2016.

[2] Z. Sun, W. Gao, B. Li, and L. Wang, Locating charging stations for electric vehicles, Transport Policy, pp. 1-7, 2018.

[3] S. Deb, K. Tammi, K. Kalita, and P. Mahanta, Review of recent trends in charging infrastructure planning for electric vehicles, Wiley Interdisciplinary Reviews: Energy and Environment, vol. 7, pp. e306, 2018.

[4] I. Capar, M. Kuby, V. Jorge Leon, and Y. Tsai, An arc cover-path-cover formulation and strategic analysis of alternative-fuel station locations, European Journal of Operational Research, vol. 277, pp. 142-151, 2013.

[5] Z. Guo, J. Deride, and Y. Fan, Infrastructure planning for fast charging stations in a competitive market, Transportation Research Part C: Emerging Technologies, vol. 68, pp. 215-227, 2016.

[6] Y. He, K. M. Kockelman, and K. A. Perrine, A Optimal locations of U.S. fast charging stations for long-distance trip completion by battery electric vehicles, Journal of Cleaner Production, vol. 214, pp. 252-461, 2019.

[7] C. Csiszár, B. Csonka, D. Foldes, E. Wirth, and T. Lovas, Urban public charging station locating method for electric vehicles based on land use approach, Journal of Transport Geography, vol. 74, pp. 173-180, 2019.

[8] Z. Yi, and M. Shirk, Data-driven optimal charging decision making fo connected and automated electric vehicles: A personal usage scenario, Transportation Research Part C: Emerging Technologies, vol. 86, pp. 37-58, 2018.

[9] Q. Liu, J. Liu, W. Le, Z. Guo, and Z. He, Data-driven intelligent location of public charging stations for electric vehicles, Journal of Cleaner Production, vol. 232, pp. 531-541, 2019

[10] H. Zhang, S. Moura, Z. Hu, and Y. Song, PEV Fast-Charging Station Siting and Sizing on Coupled Transportation and Power Networks, IEEE Transactions on Smart Grid, vol. 9, pp. 2595-2605, 2018.

[11] L. Yu, D. Kong, X. Shao, and X. Yan, A Path Planning and Navigation Control System Design for Driverless Electric Bus, IEEE Access, vol 6, pp. 53960-53975, 2018

[12] S. A. Funke, F. Sprei, T. Gnann, and P. Plotz, How much charging infrastructure do electric vehicles need? A review of the evidence and international comparison, Transportation Research Part D: Transport and Environment, vol. 77, pp. 224-242, 2019. 